\title{
Prevalência de Lesões em Corredores de Rua e Fatores Associados
}

\section{Prevalence of Injuries and Associated Factors Among Street Runners}

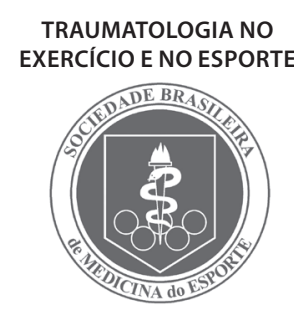

Artigo Original
Adriano Akira Ferreira Hino 1,4

Rodrigo Siqueira Reis 1,3,4

Ciro Romélio Rodriguez-Añez 3,4

Rogério César Fermino 1,2,4

1. Programa de Pós-Graduação em Educação Física, Universidade Federal do Paraná - UFPR, Brasil.

2. Mestre em Ciências do Desporto, Faculdade de Desporto da

Universidade do Porto - FADE.UP,

Portugal.

3. Professor Doutor do Curso de Educação Física, Pontifícia Universidade Católica do Paraná - PUCPR, Brasil.

4. Grupo de Pesquisa em Atividade Física e Qualidade de Vida - GPAQ / PUCPR, Brasil.

\section{Endereço para correspondência: Adriano Akira Ferreira Hino, Rua Guglielmo Marconi, 870, casa 11 , Bairro Alto - 82820-250, Curitiba, PR - Brasil. \\ E-mail: akira_manaca@yahoo.com.br}

Submetido em 29/10/2007

Versão final recebida em 06/08/2008 Aceito em 24/10/2008

\section{RESUMO}

O objetivo deste estudo foi analisar a prevalência de lesões esportivas e os fatores associados em corredores de rua por meio de um inquérito de morbidade referida. Os participantes foram selecionados por convite e por voluntariedade entre os corredores do Circuito de Ruas da Cidade de Curitiba-PR. Foram entrevistados 295 indivíduos (77,3\% homens). As prevalências foram descritas de acordo com as variáveis de estudo e a regressão de Poisson foi utilizada para testar as associações $(p<0,05)$. A prevalência de lesões nos últimos seis meses foi de 28,5\% (29,8 e 23,9\% para homens e mulheres, respectivamente). Maior prevalência de lesões foi observada na faixa etária dos 30,1 aos 45 anos (32,8\%), para aqueles com sobrepeso/obesidade (35,2\%), para mulheres que treinam mais do que $30 \mathrm{~min} / \mathrm{dia}(31,3 \%)$ e homens que treinam mais que $60 \mathrm{~min} / \mathrm{dia}$ (34,2\%). Nenhuma das variáveis investigadas apresentou associação com o relato de lesões. A maior parte dos indivíduos lesionados (60,5\%) permaneceu menos de três meses afastada, sendo maior a probabilidade dessa ocorrência (RP: 2,44; $I_{95 \%}$ : 0,99-6,06; $p=0,05$ ) para aqueles com volumes de treino entre 31 e $60 \mathrm{~min} /$ dia. Conclui-se que a prevalência de lesões foi de aproximadamente 1/3 nos homens e 1/4 nas mulheres. As variáveis investigadas não apresentaram associação com a prevalência de lesões entre os participantes de corrida de rua e a gravidade destas está associada ao volume de treino.

Palavras-chave: traumatismo em atletas, corrida, exercício, prevalência.

\begin{abstract}
The aim of this study was to analyze the prevalence of sports-related injuries and associated factors in street runners through a referred morbidity survey. Participants were selected by invitation and by volunteering among the runners from the Curitiba City Street Running Circuit during the months of September to November. 295 runners were interviewed $(77.3 \%$ men). Prevalence rates were described accordingly to the study variables and Poisson regression was employed to test associations $(p<0.05)$. The prevalence of sport injures occurred in the last six months was $28.5 \%$ (29.8\% for men and $23.9 \%$ for women). Higher prevalence rates were observed among those aged from 30.1 - 45yr (32.8\%); overweight/obese (35.2\%); women training more than $30 \mathrm{~min} /$ day (31.3\%); and men training more than $60 \mathrm{~min} /$ day (34.2\%). None of the variables analyzed were associated with self-reported sport injuries. The majority of injured subjects (60.5\%) remained less than three months away from training and those with training volume between 31 and $60 \mathrm{~min} /$ day were more likely to show this condition (prevalence ratio $=2.44 ; \mathrm{C} 195 \%=0.99-6.06 ; \mathrm{p}=0.05$ ). It is concluded that sports injuries prevalence is observed in $1 / 3$ of men and 1/4 of women. The variables investigated were not associated with prevalence of sport injuries in street runners and the injuries severity is associated with training volume.
\end{abstract}

Keywords: athletic injuries, running, exercise, prevalence.

\section{INTRODUÇÃO}

O exercício físico é usualmente associado ao bem-estar dos seus praticantes $^{(1)}$. Dentre as suas diversas manifestações, a corrida apresenta-se com uma das modalidades com grande número de adeptos, tanto pela facilidade em sua prática, como pelos benefícios para a saúde e o baixo custo ${ }^{(2)}$. Por essas e outras razões, a corrida de rua tem-se tornado popular; no entanto, os indivíduos que a praticam, seja no âmbito competitivo ou recreativo, estão expostos aos eventuais riscos associados ${ }^{(3)}$.

De acordo com Bennell e Crossley ${ }^{(4)}$ a realização de exercícios de maneira exaustiva, sem orientação ou de forma inadequada, pode contribuir para o aumento do número de lesões esportivas (LE) e estas estão associadas a fatores intrínsecos e extrínsecos. Dentre os fatores intrínsecos destacam-se a idade, o sexo, a experiência, aptidão, além de outros aspectos. Os fatores extrínsecos são o treinamento, o tipo de atividade e as condições climáticas, entre outras ${ }^{(5)}$.

Macera et al.(6), em uma revisão de literatura, relataram que entre corredores encontra-se freqüência anual de lesões de 24 a 65\%. Em um estudo onde foram entrevistados 6.596 indivíduos, 335 (5,1\%) relataram ter sofrido algum tipo de lesão durante o último mês. Também, 46\% dos homens e 15\% das mulheres referiram que as lesões estavam associadas à prática de exercícios ou esportes ${ }^{(7)}$. No Brasil, não são muitos os relatos sobre a prevalência de LE. Entre os poucos a que tivemos acesso, Domingues et al. ${ }^{(8)}$ investigaram 162 atletas do Estado São Paulo e encontraram prevalência total de 14,8\%, entre competidores de diversas modalidades esportivas. Em uma pesquisa realizada entre universitários, Conte et al. ${ }^{(9)}$ verificaram que a maior parte das LE ocorre no futebol e no atletismo, contudo, algumas características não estiveram associadas às lesões. Existe forte indício de que, em atletas de elite do atletismo brasileiro, as LE estão associadas à intensidade e ao volume de treinamento(10).

Apesar de as evidências apontarem crescente número de $L E^{(11)}$, em especial entre corredores ${ }^{(12)}$, ainda existe certa divergência sobre os fatores que podem influenciar a ocorrência desses agravos. A essa necessidade, soma-se a relativa carência de levantamentos em corredores no Brasil. Essas constatações, dificultam a realização de intervenções que possam prevenir a ocorrência de lesões nessa modalidade e também auxiliar no aumento da performance de seus praticantes ${ }^{(13)}$.

Portanto, o objetivo deste estudo foi verificar a prevalência de LE e analisar os fatores associados, em corredores participantes do circuito de corrida de rua da cidade de Curitiba-PR. 


\section{MÉTODOS}

\section{Sujeitos}

A amostra foi constituída por 295 indivíduos (228 do sexo masculino e 67 do sexo feminino) participantes do circuito de corridas de rua da cidade de Curitiba-PR no ano de 2005.

\section{Procedimentos}

A coleta de dados foi realizada por indivíduos previamente treinados para tal finalidade, entre os meses de setembro a novembro de 2005. Os sujeitos responderam de maneira espontânea às entrevistas que foram realizadas ao término de cada evento, nos locais de prova. A pesquisa foi realizada após a aprovação pelo Comitê de Ética em Pesquisa da Pontifícia Universidade Católica do Paraná (ofício 778/2005).

Para a obtenção dos dados foi utilizado um inquérito de morbidade referida (IMR), meio pelo qual é possível obter informações sobre a freqüência de agravos à saúde e seus fatores de risco ${ }^{(14)}$. O IMR foi utilizado para coletar informações pessoais, características do treinamento e descrição das lesões.

As variáveis pessoais analisadas foram o sexo, a idade, a renda familiar e o índice de massa corporal (IMC). O IMC foi dicotomizado em duas categorias: normoponderais (IMC $\leq 24,9 \mathrm{~kg} / \mathrm{m}^{2}$ ) e sobrepeso/obesidade $\left(\mathrm{IMC} \geq 25 \mathrm{~kg} / \mathrm{m}^{2}\right)$. As variáveis de treinamento investigadas foram: freqüência, duração e presença de acompanhamento profissional. Essas variáveis foram incluídas ao inquérito por ser aquelas freqüentemente associadas à prevalência de lesões ${ }^{(3,15,16)}$. Quanto às lesões, foram referidos a ocorrência e o tempo até a sua recuperação. Neste estudo, considerou-se lesão qualquer dor ou agravo que tenha limitado ou afastado a participação do atleta em treinos e/ou competições ${ }^{(11)}$ nos últimos seis meses. De acordo com Pastre et al. ${ }^{(10)}$, os dados obtidos pelo IMR com intervalo de tempo retroagindo até oito meses possuem elevada taxa de concordância com o diagnóstico profissional.

\section{Análise estatística}

Para a análise dos dados foi realizada a distribuição de freqüências das lesões relatadas de acordo com as variáveis de estudo e a medida de associação empregada foi a regressão de Poisson com significância de $5 \%$ ( $p<0,05)$. Os cálculos foram realizados por meio de software Stata versão 9.0 .

\section{RESULTADOS}

A maior parte dos indivíduos $(44,7 \%)$ se encontrava na faixa etária dos 30,1 a 45 anos, com renda > R\$1.670,00 (48,9\%). De acordo com o IMC, maior proporção da amostra foi classificada como normoponderal $(81,6 \%)$. Na sua maioria, tanto os homens quanto as mulheres despendiam até $30 \mathrm{~min} /$ dia em treinamentos $(42,6 \%)$ e com acompanhamento profissional (58,6\%) (tabela 1).

A tabela 2 apresenta a freqüência de lesões de acordo com o sexo e as variáveis analisadas. Aproximadamente $29 \%$ dos indivíduos relataram ter sofrido algum tipo de lesão nos seis meses precedentes,

Tabela 1. Medidas descritivas dos participantes da pesquisa segundo o sexo

\begin{tabular}{|c|c|c|c|c|c|c|c|}
\hline \multirow{3}{*}{ Variável } & \multirow{3}{*}{ Categorias } & \multicolumn{4}{|c|}{ Sexo } & \multirow{2}{*}{\multicolumn{2}{|c|}{ Total }} \\
\hline & & \multicolumn{2}{|c|}{ Masculino } & \multicolumn{2}{|c|}{ Feminino } & & \\
\hline & & $\mathrm{n}$ & $\%$ & $\mathrm{n}$ & $\%$ & $\mathrm{n}$ & $\%$ \\
\hline \multirow{3}{*}{ Idade } & até 30 anos & 65 & 28,6 & 17 & 25,8 & 82 & 28,0 \\
\hline & $30,1-45$ anos & 105 & 46,3 & 26 & 39,4 & 131 & 44,7 \\
\hline & $>45$ anos & 57 & 25,1 & 23 & 34,8 & 80 & 27,3 \\
\hline \multirow{3}{*}{ Renda } & $\leq \mathrm{R} \$ 425,00$ & 26 & 11,7 & 9 & 14,5 & 35 & 12,3 \\
\hline & $\mathrm{R} \$ 426,00-1.670,00$ & 87 & 39,2 & 23 & 37,1 & 110 & 38,7 \\
\hline & $>R \$ 1.670,00$ & 109 & 49,1 & 30 & 48,4 & 139 & 48,9 \\
\hline \multirow{2}{*}{ IMC } & Normoponderais & 182 & 80,2 & 58 & 86,6 & 240 & 81,6 \\
\hline & Sobrepeso/obesidade & 45 & 19,8 & 9 & 13,4 & 54 & 18,4 \\
\hline \multirow{3}{*}{ Volume de treinamento } & até $30 \mathrm{~min} /$ dia & 91 & 40,8 & 32 & 38,5 & 123 & 42,6 \\
\hline & 31-60 min/dia & 76 & 34,1 & 15 & 22,7 & 91 & 31,5 \\
\hline & $>60 \mathrm{~min} / \mathrm{dia}$ & 56 & 25,1 & 19 & 28,8 & 75 & 26,0 \\
\hline \multirow{2}{*}{ Treina com profissional } & Sim & 142 & 62,8 & 27 & 41,5 & 169 & 58,1 \\
\hline & Não & 84 & 37,2 & 38 & 58,5 & 122 & 41,9 \\
\hline
\end{tabular}

Tabela 2. Distribuição de lesões reportadas de acordo com as variáveis sociodemográficas e de treinamento

\begin{tabular}{|c|c|c|c|c|c|c|c|}
\hline \multirow{3}{*}{ Variável } & \multirow{3}{*}{ Categorias } & \multicolumn{4}{|c|}{ Sexo } & \multirow{2}{*}{\multicolumn{2}{|c|}{ Total }} \\
\hline & & \multicolumn{2}{|c|}{ Masculino } & \multicolumn{2}{|c|}{ Feminino } & & \\
\hline & & $n$ & $\%$ & $n$ & $\%$ & $\mathrm{n}$ & $\%$ \\
\hline \multirow{3}{*}{ Idade } & até 30 anos & 15 & 23,1 & 4 & 23,5 & 19 & 23,2 \\
\hline & $30,1-45$ anos & 34 & 32,4 & 9 & 34,6 & 43 & 32,8 \\
\hline & $>45$ anos & 18 & 31,6 & 3 & 13,0 & 21 & 26,3 \\
\hline \multirow{3}{*}{ Renda } & $\leq \mathrm{R} \$ 425,00$ & 8 & 30,8 & 4 & 44,4 & 12 & 34,3 \\
\hline & $R \$ 426,00-1.670,00$ & 22 & 25,3 & 1 & 4,3 & 23 & 20,9 \\
\hline & $>R \$ 1.670,00$ & 38 & 34,9 & 11 & 36,7 & 49 & 35,3 \\
\hline \multirow{2}{*}{ IMC } & Normoponderais & 52 & 28,6 & 13 & 22,4 & 65 & 27,1 \\
\hline & Sobrepeso/obesidade & 16 & 35,6 & 3 & 33,3 & 19 & 35,2 \\
\hline \multirow{3}{*}{ Volume de treinamento } & até $30 \mathrm{~min} / \mathrm{dia}$ & 24 & 26,4 & 10 & 31,3 & 34 & 27,6 \\
\hline & $31-60 \mathrm{~min} / \mathrm{dia}$ & 26 & 34,2 & 12 & 13,3 & 38 & 30,8 \\
\hline & $>60 \mathrm{~min} / \mathrm{dia}$ & 18 & 32,1 & 4 & 21,1 & 22 & 29,3 \\
\hline \multirow{2}{*}{ Treina com profissional } & $\operatorname{sim}$ & 29 & 34,5 & 9 & 23,7 & 38 & 31,1 \\
\hline & Não & 39 & 27,5 & 6 & 22,2 & 45 & 24,2 \\
\hline Sofreram lesão & & 68 & 29,8 & 16 & 23,9 & 84 & 28,5 \\
\hline
\end{tabular}


sendo a maior prevalência verificada entre os homens (29,8\%). Dentre as características pessoais, maior prevalência foi observada na faixa etária de 30,1 a 45 anos (32,8\%) para ambos os sexos, sendo menor entre mulheres $>45$ anos (13\%) do que entre os homens da mesma faixa etária (31,6\%). A prevalência foi similar entre as categorias de renda $\leq R \$ 425,00$ e $>R \$ 1.670,00$ e menor na categoria $R \$ 426,00-1.670,00$. A freqüência de lesões foi superior nas mulheres com renda até $R \$ 425,00$ $(44,4 \%)$ quando comparadas com os homens (30,8\%). Em relação ao IMC observou-se maior prevalência para aqueles com sobrepeso/obesidade $(35,2 \%)$, sendo a distribuição similar entre os sexos. Entre as características de treinamento, se considerarmos homens e mulheres, a prevalência de lesões foi similar entre as categorias de volume. No entanto, verificou-se resultado inverso entre os sexos, com maior prevalência entre as mulheres que treinavam até $30 \mathrm{~min} /$ dia $(31,3 \%)$ e entre os homens que treinavam de 31 a $60 \mathrm{~min} / \mathrm{dia}$ (34,2\%).

Na tabela 3 são apresentados os resultados das medidas de associação entre as variáveis de estudo e a prevalência de lesões. Embora não tenha sido verificada associação entre a prevalência de lesões e as variáveis investigadas, um efeito elevado foi observado para aqueles com sobrepeso/obesidade (RP:1,30; IC $95 \%: 0,78-2,17 ; p=0,32$ ) quando comparados com os normoponderais e ainda para os corredores com idade entre 30,1 e 45 anos (RP:1,42; IC $95 \%$ : 0,83-2,43; $p=0,21$ ).

Tabela 3. Valores de razão de prevalência (RP) e intervalo de confiança a 95\% (IC95\%) para as prevalências de lesões de acordo com as variáveis de estudo

\begin{tabular}{|c|c|c|c|}
\hline \multirow{2}{*}{ Variáveis } & \multirow{2}{*}{ Categorias } & \multicolumn{2}{|c|}{ Masculino } \\
\hline & & RP & $\mathrm{IC}_{95 \%}$ \\
\hline & Masculino & 1 & \\
\hline & Feminino & 0,80 & $0,46-1,38$ \\
\hline \multirow{3}{*}{ Idade } & até 30 anos & 1 & \\
\hline & $30,1-45$ anos & 1,42 & $0,83-2,43$ \\
\hline & $>45$ anos & 1,13 & $0,61-2,11$ \\
\hline \multirow{3}{*}{ Renda } & $\leq R \$ 425,00$ & 1 & \\
\hline & $R \$ 426,00-1.670,00$ & 0,61 & $0,30-1,23$ \\
\hline & $>\mathrm{R} \$ 1.670,00$ & 1,03 & $0,78-2,17$ \\
\hline \multirow{2}{*}{ IMC } & Normoponderais & 1 & \\
\hline & Sobrepeso/obesidade & 1,30 & $0,78-2,17$ \\
\hline \multirow{3}{*}{$\begin{array}{l}\text { Volume de } \\
\text { treinamento }\end{array}$} & até $30 \mathrm{~min} /$ dia & 1 & \\
\hline & 31-60 min/dia & 1,11 & $0,68-1,84$ \\
\hline & $>60 \mathrm{~min} / \mathrm{dia}$ & 1,06 & $0,62-1,81$ \\
\hline \multirow{2}{*}{$\begin{array}{l}\text { Treina com } \\
\text { profissional }\end{array}$} & $\operatorname{sim}$ & 1 & \\
\hline & Não & 0,85 & $0,56-1,32$ \\
\hline
\end{tabular}

Em relação à duração da lesão, a maior parte dos indivíduos (60,5\%) apresentou afastamento inferior a três meses. Maior proporção de muIheres permaneceu afastada por esse período de tempo (86,7 versus 54,5\%). Não foram observadas discrepâncias nas prevalências de afastamento superior a três meses nas demais variáveis demográficas. No entanto, quando os aspectos de treinamento foram analisados como variáveis independentes (tabela 4), observou-se maior probabilidade de afastamento (superior a três meses) entre os indivíduos que apresentaram volumes de treinamento de 31 a $60 \mathrm{~min} / \mathrm{dia}$ (RP: 2,44; IC $\mathrm{C}_{95 \%}$ : 0,99-6,06; $p=0,05)$, comparados com aqueles que treinavam com menor volume (até $30 \mathrm{~min} / \mathrm{dia}$ ). Contudo, quando a análise foi ajustada ao sexo, à idade e ao IMC, a associação não foi significativa. O treinamento acompanhado por profissionais não foi associado a menor risco de afastamento provocado por lesões inferior a três meses.

\section{DISCUSSÃO}

O entendimento dos fatores associados a maior ocorrência de LE é de grande importância para que possíveis medidas preventivas sejam realiza-
Tabela 4. Valores de razão de prevalência (RP) brutos e ajustados (sexo, idade e IMC) e respectivos intervalos de confiança a 95\% (IC95\%) para as prevalências de afastamentos inferiores a três meses de acordo com os aspectos do treinamento

\begin{tabular}{c|c|c|c|c|c}
\hline Variável & Categorias & $\mathbf{R P}$ & $\mathbf{I C}_{\mathbf{9 5} \%}$ & $\mathbf{R P}_{\text {ajust }}$ & $\mathbf{I C}_{\mathbf{9 5} \%}$ \\
\hline \multirow{2}{*}{$\begin{array}{c}\text { Volume de } \\
\text { treinamento }\end{array}$} & até $30 \mathrm{~min} / \mathrm{dia}$ & 1 & & 1 & \\
\cline { 2 - 6 } & $31-60 \mathrm{~min} / \mathrm{dia}$ & $2,44^{*}$ & $0,99-6,06$ & 2,09 & $0,82-5,33$ \\
\cline { 2 - 6 } & $>60 \mathrm{~min} / \mathrm{dia}$ & 2,47 & $0,96-6,37$ & 2,06 & $0,82-5,90$ \\
\hline \multirow{2}{*}{$\begin{array}{c}\text { Treina com } \\
\text { profissional }\end{array}$} & $\mathrm{Sim}$ & 1 & & 1 & \\
\cline { 2 - 7 } & $\mathrm{Não}$ & 0,93 & $0,46-1,86$ & 0,88 & $0,42-1,90$ \\
\hline
\end{tabular}

${ }^{*} p \leq 0,05$

das com maior efetividade. O presente estudo buscou identificar a prevalência de lesões (e os fatores associados) em uma amostra de corredores de rua. Nas buscas realizadas pelos autores em bases de dados nacionais e da América Latina, não foram identificadas pesquisas dessa natureza com tal população, sendo este estudo, portanto, original nesse aspecto. Na presente pesquisa a prevalência de lesões relatadas entre os corredores (28,5\%) é similar àquela apresentada em outros estudos. Em uma pesquisa realizada por Taunton et al. ${ }^{(17)}, 884$ corredores foram acompanhados durante um programa de 13 semanas de treinamento, período em que 29,5\% deles relataram alguma lesão. Em um estudo ${ }^{(18)}$ realizado com pacientes de uma clínica de medicina preventiva, 5.028 homens e 1.285 mulheres foram avaliados. Os resultados mostraram que 20,9\% dos homens e 20,3\% das muIheres, sofreram algum tipo de lesão muscular nos 12 meses antecedentes à pesquisa. Ainda, os indivíduos fisicamente ativos que praticavam corridas regulares relataram freqüência de lesão entre 23,2\% e 24,7\% (mulheres e homens, respectivamente). Em um inquérito realizado por telefone no qual foram entrevistados 6.596 indivíduos, 32,5\% dos participantes relataram ter sofrido algum tipo de lesão no último mês devido à participação em esportes ou exercícios físicos. A maior ocorrência foi no sexo masculino (46,4 versus 13,5\%), porém, o tipo de esporte ou exercício que ocasionou a lesão não foi elucidado ${ }^{(7)}$. Um estudo envolvendo militares encontrou, após 12 semanas de treinamento, freqüência total de lesões de 28\%(19). Embora os dados da literatura pareçam corroborar os achados do presente estudo, a comparação é dificultada pela diferença nos critérios empregados para definir "lesão" utilizada nas diversas pesquisas, assim como pela variação entre os períodos referentes ao histórico da lesão.

O decréscimo observado na freqüência de lesões com o aumento da idade é corroborado por estudos que analisaram a participação em esportes em geral(7). Um estudo realizado por Hootman et al. ${ }^{(12)}$ apresentou diminuição na prevalência de lesões a partir dos 60 anos de idade, sendo que os sujeitos entre 40-60 anos são mais acometidos quando comparados com aqueles com idade inferior a 40 anos. Essas evidências sugerem que o decréscimo da ocorrência de lesões observado com o aumento da idade deve-se à redução na freqüência e intensidade da atividade física com o decorrer do envelhecimento.

A relação entre o IMC e a ocorrência de lesões tem sido discutida na literatura. Enquanto alguns estudos não apresentam qualquer associação(17), outros demonstraram que o IMC (elevado ou não) tem sido associado com as $L E^{(15)}$. No presente estudo, menor freqüência de lesões foi verificada entre os indivíduos normoponderais em relação ao grupo classificado com sobrepeso/obesidade. A classificação em um IMC mais elevado tem apresentado relação inversa com a prevalência de lesões ${ }^{(10,17)}$. Hootman et al. ${ }^{(12)}$ encontraram maior prevalência de lesões entre indivíduos normoponderais (IMC $\left.<25 \mathrm{~kg} / \mathrm{m}^{2}\right)$. De fato. alguns estudos não têm demonstrado relação entre IMC e a prevalência de lesões. Essa divergência entre as evidências parece sugerir que essa relação é inconclusiva.

Ao se tratar de lesões relacionadas ao "excesso de uso" (overuse) em corredores, parece existir um consenso entre os pesquisadores de que as variáveis relacionadas ao treino são as causas de grande parte das lesões ${ }^{(20)}$. Também foi verificado que a corrida é uma das atividades 
em que as lesões mais ocorrem quando comparando a prática esportiva e de caminhadas ${ }^{(12)}$, em especial nos membros inferiores ${ }^{(20,21)}$. No presente estudo, quando ambos os sexos são agrupados, verifica-se que o aumento no volume de treinamento não é acompanhado pelo aumento na freqüência de lesões, diferentemente do relatado na literatura. Hootman et al. ${ }^{(12)}$ concluem que existe aumento do risco de lesão musculoesquelética entre corredores conforme o incremento do volume semanal de treinamento. Os corredores que treinavam mais de 3,75 horas/semana possuíam 2,38 (IC $95 \%$ : 1,72-3,29) mais probabilidade de sofrer lesões quando comparados com os indivíduos que treinavam menos que 1,25 hora/semana. Shaw et el. ${ }^{(22)}$ encontraram associação entre as horas de treino e a ocorrência de lesão em praticantes de triátlon. De acordo com os autores, os triatletas com baixos e elevados volumes de treino possuem maior probabilidade de sofrer algum tipo de lesão em relação aos que treinam com volumes moderados. No entanto, os resultados da tabela 3 demonstram que, entre os homens, verifica-se que a menor freqüência de lesões encontra-se entre aqueles que treinam até $30 \mathrm{~min} / \mathrm{dia}(26,4 \%)$. Nas mulheres, a maior prevalência foi observada entre as que treinavam até $30 \mathrm{~min} /$ dia (31,3\%). Um dos aspectos que podem influenciar a ocorrência das lesões é a intensidade do treinamento ${ }^{(23)}$. Contudo, essa variável não foi controlada. O que poderia, em parte, explicar as discrepâncias observadas entre os sexos.

Diante dos resultados, parece que, para as mulheres, a presença de acompanhamento profissional durante os treinamentos tende a favorecer a ocorrência de lesões, pois 60\% relataram terem sofrido algum agravo. Entre os homens a prevalência foi inferior (42,6\%). Observações realizadas a partir de estudos clínicos estimaram que $\approx 60 \%$ das lesões em corredores podem ser atribuídos a erros de treinamento(21). Porém, mesmo a exposição de lesões sendo maior durante a fase de treinamento, a probabilidade de alguma lesão ocorrer durante a competição é aproximadamente quatro vezes maior ${ }^{(24)}$.

Quando a prática do exercício possui um foco sobre o desempenho em competições, verifica-se maior ocorrência de lesões ${ }^{(25)}$, mesmo quando supervisionado por profissionais ${ }^{(26)}$. Em competidores de atletismo participantes de campeonatos nacionais e internacionais, a freqüência de atletas acometidos por lesões variou de 75 a 100\%, dependendo da prova praticada ${ }^{(10)}$. Mesmo em treinamentos supervisionados por profissionais e com programas específicos para a prevenção de lesões, a freqüência desse agravo pode chegar a $\approx 30 \%{ }^{(17)}$. No presente estudo, o maior percentual de acometimentos observados em atletas que treinavam com acompanhamento profissional pode, de alguma maneira, ser atribuído aos objetivos de desempenho estipulados pelos treinadores.

\section{REFERÊNCIAS BIBLIOGRÁFICAS}

1. Haskell WL, Lee IM, Pate RR, Powell KE, Blair SN, Franklin BA, et al. Physical Activity and Public Health: Updated Recommendation for Adults from the American College of Sports Medicine and the American Heart Association. Med Sci Sports Exerc. 2007:39(8):1423-34.

2. Paluska SA. An overview of hip injuries in running. Sports Med. 2005;35(11):991-1014.

3. Neely FG. Biomechanical risk factors for exercise-related lower limb injuries. Sports Med. 1998;26(6):395-413.

4. Bennell KJ, Crossley K. Musculoskeletal injuries in track and field: incidence, distribution and risk factors. Aust J Sci Med Sport. 1996;28(3):69-75.

5. Gissane C, White J, Kerr K, Jennings D. An operational model to investigate contact sports injuries. Med Sci Sports Exerc. 2001;33(12):1999-2003.

6. Macera CA, Pate RR, Powell KE, Jackson KL, Kendrick JS, Craven TE. Predicting lower-extremity injuries among habitual runners. Arch intern med. 1989 nov;149(11):2565-8.

7. Uitenbroek DG. Sports, exercise, and other causes of injuries: results of a population survey. Res $Q$ Exerc Sport. 1996;67(4):380-5.

8. Domingues SPT, Conte M, Más EF, Ramalho LCB, Godoi VJ, Teixeira LFM, et al. Implicações do nível de aptidão física na gênese de lesões esportivas. Rev Bras Cineantropom Desempenho Hum. 2005;7:29-35.

9. Conte M, Júnior EM, Arruda LV, Chalita S, Gonçalves A. Exploração de fatores de risco de lesões desportivas entre universitários de educação física: estudo a partir de estudantes de Sorocaba/SP. Rev Bras Med Esporte. 2002;8:151-6.

10. Pastre CM, Filho GC, Monteiro HL, Júnior JN, Padovani CR. Lesões desportivas na elite do atletismo brasileiro: estudo a partir de morbidade referida. Rev Bras Med Esporte. 2005;11:43-7.

11. Lun V, Meeuwisse WH, Stergiou P, Stefanyshyn D. Relation between running injury and static lowe limb alignment in recreational runners. Br J Sports Med. 2004;38(5):576-80.

12. Hootman JM, Macera CA, Ainsworth BE, Martin M, Addy CL, Blair SN. Association among physical activity level, cardiorespiratory fitness, and risk of musculoskeletal injury. Am J Epidemiol. 2001;154(3):251-8.
Embora não tenham sido evidenciadas características que estivessem notadamente associadas à ocorrência de lesões entre os participantes do estudo, a severidade das lesões diferiu entre os mesmos. A prevalência de afastamento superior a três meses foi maior entre aqueles com volumes de treinamento entre 31 e $60 \mathrm{~min} /$ dia, correspondendo ao verificado em outros estudos ${ }^{(12,22)}$. No entanto, tal associação não é mantida quando o sexo, idade e IMC são considerados, o que pode ser explicado, em parte, pela maior prevalência observada entre as mulheres participantes.

Os resultados do presente estudo são preocupantes, uma vez que a promoção da atividade física possui como premissa o desenvolvimento de um estilo de vida mais saudável. Segundo Uitenbroek ${ }^{(7)}$, as lesões podem trazer conseqüências psicológicas e sociais e possivelmente uma imagem desfavorável à realização de atividades físicas. Por essa razão, as pesquisas que buscam identificar fatores associados à ocorrência de lesões devem ser desenvolvidas para que o treinamento seja realizado de maneira segura. Essa recomendação tem especial significado, uma vez que a popularidade das corridas de rua tem aumentado, expondo, dessa maneira, maior número de indivíduos.

Dentre as limitações dessa pesquisa destacam-se o problema de causalidade reversa típico de estudos transversais, não permitindo assim determinar a direção exata da relação causal. Também se deve considerar que o IMR utilizado para determinar a presença de lesões, embora seja um método amplamente aceito e empregado, possui menor especificidade do que o diagnóstico clínico.

Conclui-se que entre os corredores do sexo masculino, aproximadamente 1/3 dos indivíduos relatou ter sofrido algum tipo de lesão nos seis meses antecedentes à pesquisa. Desses, quase metade permaneceu lesionada por no mínimo três meses. Entre as mulheres aproximadamente $1 / 4$ relatou ter sofrido lesão, das quais uma maior proporção delas permaneceu lesionada pelo menos um mês. Dentre as variáveis investigadas, nenhuma apresentou associação com a prevalência de lesões entre os participantes de corrida de rua.

\section{AGRADECIMENTOS}

Aos membros do Grupo de Pesquisa em Atividade Física e Qualidade de Vida - GPAQ/PUCPR, pela colaboração na coleta de dados. Também, às pertinentes sugestões de ambos os revisores anônimos, as quais colaboraram para melhor compreensão do conteúdo deste artigo.

Todos os autores declararam não haver qualquer potencial conflito de interesses referente a este artigo.
13. Moreira P, Gentil D, Oliveira CD. Prevalência de lesões na temporada 2002 da Seleção Brasileira Masculina de Basquete. Rev Bras Med Esporte. 2003;5:258-62.

14. Pereira MG. Epidemiologia: teoria e prática. Rio de Janeiro; Guanabara Koogan; 1995.

15. Knapik JJ, Sharp MA, Canham-Chervak M, Hauret K, Patton JF, Jones BH. Risk factors for training-related injuries among men and women in basic combat training. Med Sci Sports Exerc. 2001;33(6):946-54

16. Jones BH, Knapik JJ. Physical training and exercise-related injuries. Surveillance, research and injury prevention in military populations. Sports Med. 1999;27(2):111-25.

17. Taunton JE, Ryan MB, Clement DB, McKenzie DC, Lloyd-Smith DR, Zumbo BD. A prospective study of running injuries: the vancouver sun run "in training" clinics. Br J Sports Med. 2003;37(3):239-44.

18. Hootman JM, Macera CA, Ainsworth BE, Addy CL, Martin M, Blair SN. Epidemiology of musculoskeletal injuries among sedentary and physically active adults. Med Sci Sports Exerc. 2002;34(5):838-44

19. Rosendal L, Langberg H, Skov-Jensen A, Kjaer M. Incidence of injury and physical performance adaptations during military training. Clin J Sport Med. 2003;13(3):157-63.

20. Hreljac A, Marshall RN, Hume PA. Evaluation of lower extremity overuse injury potential in runners, Med Sci Sports Exerc. 2000;32(9):1635-41.

21. Hreljac A. Impact and overuse injuries in runners. Med Sci Sports Exerc. 2004;36(5):845-9.

22. Shaw $T$, Howat $P$, Trainor M, Maycock B. Training patterns and sports injuries in triathletes. J SC Med Sport. 2004;7(4):446-50

23. Sallade JR, Koch S. Training errors in long distance running. J Athl Train. 1992;27(1):50-3.

24. Zemper ED. Track and field injuries. Med Sport Sci. 2005;48:138-51.

25. Egermann M, Brocai D, Lill CA, Schmitt H. Analysis of injuries in long-distance triathletes. Int J Sports Med. 2003;24(4):271-6.

26. Taunton JE, Ryan MB, Clement DB, McKenzie DC, Lloyd-Smith DR, Zumbo BD. A retrospective case-control analysis of 2002 running injuries. Br J Sports Med. 2002;36(2):95-101. 